\title{
The Technological Development of Different Generations and Reactor Concepts
}

\author{
David Reinberger, Amela Ajanovic, and Reinhard Haas ${ }^{1}$
}

\begin{abstract}
This chapter documents the technical development of different generations of nuclear power plants and provides an outlook for possible future concepts and their market prospects. The objective is to assess whether there is really significant technological progress on the horizon and whether these "new" concepts have prospects to become cost-effective. A major conclusion is that most of the so-called Generation IV concepts have already been discussed in the 1950s. At that time, they have not been pursued further due to problems such as costs, limiting factors in material properties and problems in appropriately controlling the fission processes. Yet, since about 2000 a modest revival of the discussion on these concepts is observed, obviously mainly motivated by securing the flow of public money for nuclear research and the broad recognition that with present reactor concepts the nuclear industry will not succeed.
\end{abstract}

1 Reinhard Haas, Technische Universität Wien, Austria, haas@eeg.tuwien.ac.at; Amela Ajanovic, Technische Universität Wien, Austria, ajanovic@eeg.tuwien.ac.at; David Reinberger, Magistrat Stadt Wien, Austria, david.reinberger@wien.gv.at

(C) The Author(s) 2019

R. Haas et al. (Eds.), The Technological and Economic Future of Nuclear Power, Energiepolitik und Klimaschutz. Energy Policy 


\section{Introduction}

Since the first nuclear power plants (NPP) were built in the 1950s, several technical developments have taken place. This chapter documents the changes in various generations of nuclear power plant designs, their major features and differences and to analyze technological problems arising mainly with respect to safety. In addition, we focus on the prospects of so-called Generation IV (GEN IV) reactors.

According to the WNISR (2018), as of mid-2018, world-wide 413 nuclear power reactors were operating in 31 countries. The design, size and age of these reactors vary widely. Some of them are from the 1960's, directly derived from designs for military purposes. Over two thirds (69\%) of the reactors in operation are Pressurized Water Reactors (PWR), 13 \% Boiling Water Reactors (BWR), 11 \% Pressurized Heavy Water Reactor (PHWR). The majority of them is older than 30 years.

Apart from the basic features (i.e. type of coolant, moderator material, working temperature, thermal/fast neutrons), the designs are categorized by "generations" (GEN); i.e. Generation I, II, III, III+, and IV. For GEN III and GEN III+, there are no clear definitions of which reactor design fits to which generation. It can be seen as an indicative classification. The major motivation for the nuclear industry for developing new reactor concepts are: (i) high current costs; (ii) low current fuel use efficiency; (iii) limited available uranium-resources; (iv) problems with refueling schedule; (v) problems concerning safety and waste management.

\section{$2 \quad$ Historical development of reactor concepts}

This sub-chapter provides a concise overview and critical review of the history of reactor concepts from GEN-I to GEN-III+, their advantages and their weaknesses. In addition, an outlook on GEN-IV reactor types is given. It is claimed that they are radically different designs compared to those in use today. If ever, they are expected to enter markets in 20 to 40 years.

Today three generations of nuclear reactors are operating worldwide. (Goldberg et. al (2011)). Figure 1 shows when specific concepts entered the market or are expected to do so. Today, both GEN II and GEN III plants are still being planned, built and used. Even GEN I plants are still in operation despite their lack of safety features. 


\section{Generation I}

GEN I plants are the early prototypes and power reactors from the 1950s and 1960s that were launched to generate nuclear power for commercial (civilian) purposes. They were deployed directly from military applications for commercial purposes without any additional safety devices. At least some of them were clearly built for dual-use (civil and military) purpose. GEN-I plants did not have any (active or passive) dedicated safety devices.

Examples of GEN I are the Soviet AMB in Belojarsk or the AM-1 in Obninsk (both are graphite moderated types which have advantageous properties for the plutonium production), the UK's “Magnox" gas-cooled reactor - e.g. Calder Hall-1 (1956-2003), Wylfa (1959-2012) (also Graphite moderated) - and the US reactors of the first generation such as Shippingport (1957-1982) in Pennsylvania (PWR) and Dresden-1 (BWR) (1960-1978) in Illinois. Most of them have already been shut down.

The last European remaining commercial Gen I plant, the Wylfa Nuclear Power Station in Wales, has been scheduled for closure in 2012, and finally was shut down in December 2015. In Pakistan the last GEN-I plant - a CANDU-137 - is still operational at Karachi.

\section{Generation II}

GEN II systems began operation in the late 1960s and comprise the vast majority of today's operating reactors ordered from the mid-60s to the 2010s. They are designed for a typical average operational lifetime of 40 years. The major difference to the GEN-I reactors are dedicated active safety designs and that they were in principle designed for civil use only.

GEN-II reactors are typically light water reactors (LWRs) even though there are heavy water designs too. They use safety features involving electrical or mechanical operations that are initiated automatically and, in many cases, can be initiated by the operators.

Figure 1 depicts the evolution of different generations of nuclear power reactors: GEN I, II and III in operation, GEN III+ as near-term deployment, and finally the GEN IV expected to be deployed not before 2030. 


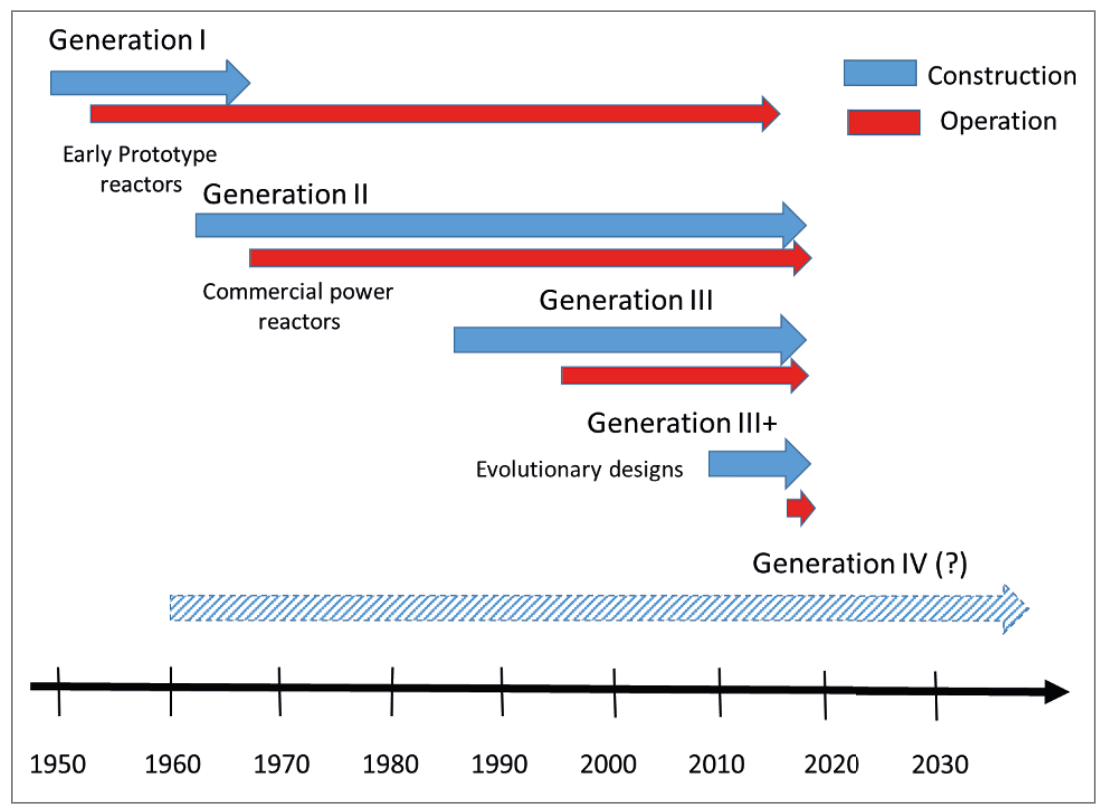

Fig. 1 The evolution of different generations of nuclear power reactors

Most of the GEN II plants still in operation in the Western countries were manufactured by Westinghouse, Framatome, and General Electric (GE). In the following section, the most important GEN-II systems are described in more detail.

\section{Pressurized water reactors}

The pressurized water reactor (PWR) was developed from the reactors used to propel submarines. In contrast to submarine reactors, which use high-enriched uranium, PWRs employ low-enriched uranium. They are water cooled and moderated. The power density of PWRs can be relatively high $\left(100 \mathrm{MW} / \mathrm{m}^{3}\right)$ due to the effectiveness of the heat removal. 


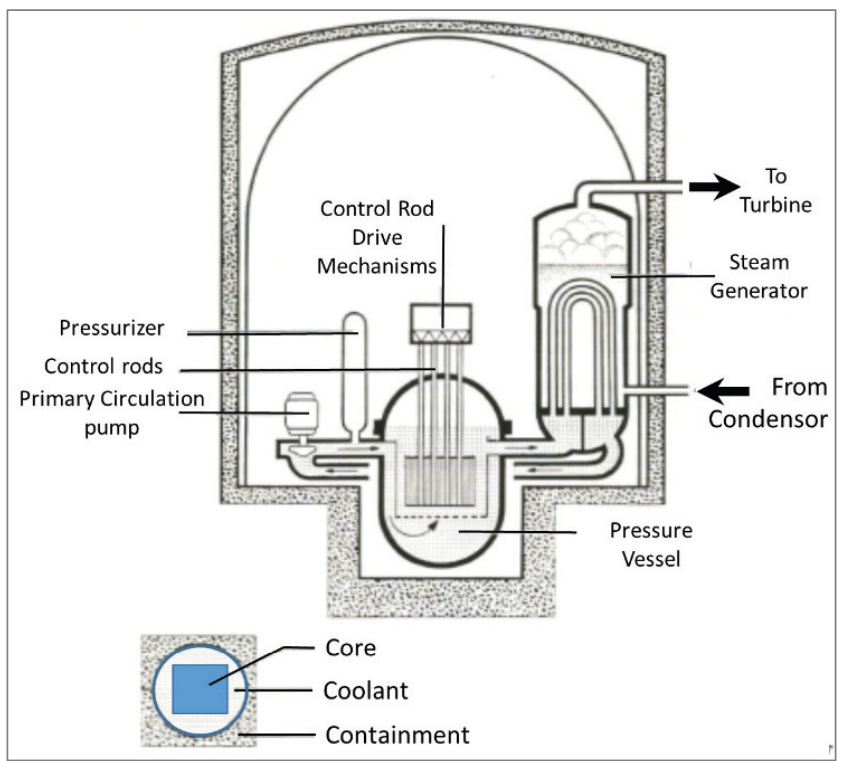

Fig. 2 Description of a pressurized water reactor (based on Greenpeace (2005))

Their primary circuit is characterized by high pressure $(\sim 15 \mathrm{M} \mathrm{Pa})$ and high temperature $\left(\sim 600^{\circ} \mathrm{F} / \sim 300^{\circ} \mathrm{C}\right)$. Safety shall be guaranteed by different systems to control the power output and to cool the core and the barrier system, consisting subsequently of the material structure of the uranium-pellets, the cladding of the fuel rods, the reactor pressure vessel (RPV) itself, the concrete structure around the RPV and the containment, which encloses the primary circuit.

The steam generators, the link between the primary and secondary circuits, are a potential source for radioactivity leaking to the outside. Due to the high power density and the correspondingly high decay heat generated after shutdown, PWRs depend heavily on the availability of the cooling system. The active systems depend on a continuous electricity supply. Even when emergency diesels, redundant grid connection and batteries are installed, station blackouts represent a serious risk, which i. e. in Fukushima led to the destruction of all safety barriers.

Safety systems are usually redundant (i.e. more components are provided for a task than needed). However, redundancy becomes useless if a so-called 'common cause-failure' like flooding or fire disables all parallel trains of a safety system. In principle, there is a continuous trend worldwide towards increasing automation in 
nuclear power plants' safety, which potentially can reduce the hazards of human error. There is also a trend to replace original analog $I \& C$ with digital systems. The implications on safety of this shift are discussed still controversial.

\section{Boiling Water Reactors}

The boiling water reactor (BWR) was developed from the PWR, in an attempt to achieve greater simplicity of design and higher thermal efficiency by using a single circuit and by generating steam within the reactor core. As for PWRs, water acts as moderator and coolant. The result is a reactor that still exhibits most of the hazardous features of the PWR, while introducing a number of new problems.

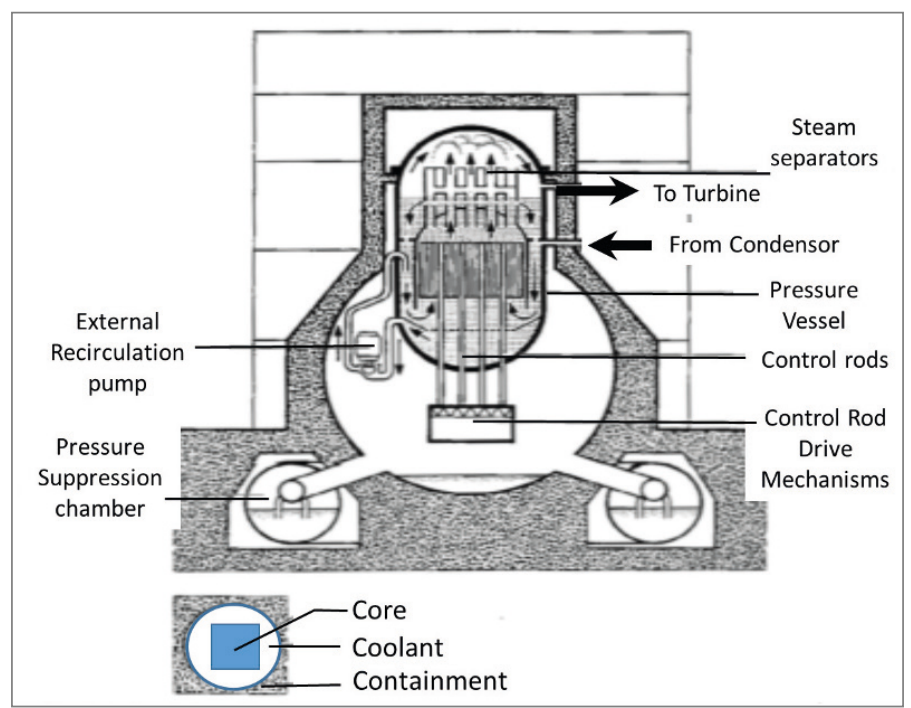

Fig. 3 Description of a boiling water reactor (based on Greenpeace (2005))

BWRs have lower power density $\left(\sim 50 \mathrm{MW} / \mathrm{m}^{3}\right)$ in the core as well as lower pressure and lower temperature in their cooling circuit than a PWR. The uranium inventory in the core is higher than in PWRs.

The primary circuit of a BWR passes outside the reactor containment. The radioactive steam is directly used to power the turbine(s). Neutron fluxes are considerably lower (by a factor of 10) than in a PWR, leading to significantly less 
neutron induced aging of the reactor materials. On the other hand, the vessel is much larger; longitudinal welds may be required, whereas there are only circumferential welds in a PWR vessel. There is also a much more complicated inner structure, as well as many penetrations at the bottom. Like a PWR, a BWR depends heavily on fast and reliable active safety systems, while the plumbing of the BWR's emergency core cooling system is much more complex. Control rod injection is - against gravity - from underneath the pressure vessel, Regulating the operation of a BWR is generally more complex than in a PWR. Under certain circumstances, the collapse of so-called steam voids in the core can lead to increasing reactivity and thus increasing power during an accident.

\section{Generation III and Generation III +}

GEN III designs began to emerge in the mid-1980s onwards, based on learning from the Three Mile Island and Chernobyl accidents. A number of 'evolutionary' GEN III designs were developed from GEN II reactor types without any drastic changes. According to the World Nuclear Association (WNA), GEN III reactors are characterized by improvements in the following areas (WNA 2004):

- a more standardised design for each type to expedite licensing, reduce capital cost and reduce construction time,

- simpler and more rugged design, making them easier to operate and less vulnerable to operational upsets,

- longer operating life - typically 60 years,

- reduced possibility of core melt accidents,

- better fuel technology, higher thermal efficiency, higher burn-up to reduce fuel use and amount of waste,

- modularized construction.

The most significant improvement of GEN III systems is the incorporation of passive safety features in some designs, which do not require active controls or operator intervention but instead rely on gravity or natural convection to mitigate the impact of abnormal events. Passive systems do not work under any circumstances. They may not relay on functioning of some technical systems but they relay on certain external (uncontrollable) parameters under which the desired physical/chemical process works. 
Different concepts bearing the labels GEN III and GEN III+ are in various stages of development and implementation today. In the following, the most important examples as mentioned by WNA (2004) and the International Atomic Energy Agency (IAEA, 2004) are provided. Usually, the concepts are classified into two categories: Large designs $>700$ MWe and medium designs $<700$ MWe.

\section{Pressurized Water Reactors}

The principal large designs are APWR (Mitsubishi Heavy Industries (MHI)/ Westinghouse), APWR+ (MHI), EPR (AREVA), AP-1000 (Westinghouse), KSNP+ and APR-1400 (Korean Industry) and the CNP-1000 (China National Nuclear Corporation). Regarding the Russian VVERs, an advanced VVER-1000 has been developed by Atomenergoproject and Gidropress.

The main small- and medium-size advanced PWR designs are the AP-600 (Westinghouse) and the VVER-640 (Atomenergoproject and Gidropress).

\section{Boiling Water Reactors}

The main large concepts are the ABWR, (Hitachi, Toshiba, GE), the BWR 90+ (Westinghouse Atom of Sweden), the SWR-1000 (Framatome ANP) and the ESBWR (GE). The HSBWR and HABWR (Hitachi) are small- and medium-sized advanced BWR concepts.

Three ABWRs were already operating in Japan: Two at Kashiwazaki-Kariwa since 1996, a third started operating in 2004. As of the end of 2018, none of them is operational due to shutdown following earthquakes in July 2007 and March 2011.

\section{Generation III+}

Generation III+ reactors are slightly modified GEN III designs initiated in the late 1990s, when the nuclear industry began to promote a 'nuclear renaissance'. These should solve three key problems: safety, cost and buildability. The central claim was based on the premise that the existing designs have become too complex and expensive due to new safety systems.

Construction costs of US $\$ 1,000 / \mathrm{kW}$ were forecast, a level that would make nuclear competitive with gas, and construction times of four years or less were expected (see previous WNISRs). The promise that nuclear power could be the cheapest option reignited interest in nuclear power in a number of key markets.

What are the lessons learned today? Regarding the claim that designs would be easier to build, 20 years since a 'nuclear renaissance' has been announced, only a handful of GEN III+ reactors - four AP-1000, one EPR - have started operating, in 2018 , in China. Standardization did not take place, and the introduction of mod- 
ularized design seems to have simply shifted the quality issues from construction sites to module factories.

By end of 2018, an additional 11 reactors claiming to meet GEN III+ criteria whatever they are - were under construction: six AES-2006, three EPRs and two AP-1000, all of them years behind schedule and significantly over budget. The construction of two AP-1000s in the U.S., at the VC Summer site in South Carolina, was abandoned in 2017 after builder Westinghouse went bankrupt.

\section{$4 \quad$ Small Modular Reactors (SMRs)}

As nuclear power generation has been established in the 1950s, the capacity of reactor units has grown from $60 \mathrm{MWe}$ to more than $1400 \mathrm{MWe}$, with corresponding economies of scale in operation. However, these large plants operating today set a high and expensive standard for safety.

Several firms are working on designs that are smaller in scale than the current GEN III designs. A basic feature of these plants is that they would make use of modular construction techniques. Small components shall be assembled in a factory environment (offsite or onsite) into structural modules weighing up to 1,000 tonnes. The idea is that these new reactors-at a $300 \mathrm{MW}$ scalable, smaller than a rail car and one-tenth the cost of a big plant-could be built quickly.

The driving forces for SMRs are the reduction of investment per unit and the need for integration into smaller grids in developing countries. However, there are serious doubts concerning these prospects. Furthermore, the opinion that the only way to make nuclear power cost competitive is the use of small modules is not shared by all nuclear industry experts. The reason is - see above - the achieved economies-of-scale with respect to LWR. Capital construction costs (price per unit of electric capacity, or $\$ / \mathrm{kWe}$ ) of a nuclear reactor decrease with size, but the economy of scale applies only if reactors are of a very similar design, as has historically been the case.

The design characteristics of SMRs, however, are significantly different from those of large reactors. SMRs approach the economies of scale problem by achieving significant cost savings elsewhere leading to significant learning-by-doing efficiencies. For example, SMR designs seek to streamline safety and safeguard requirements by replacing (at least some) security guards with concrete security barriers and/ or by building underground, streamlining the requirements for operators and the emergency planning zone requirements. 
The new designs do stretch out refuelling schedules, from 18 months to possibly $3-5$ years and potentially to as long as 10 years.

\section{Generation IV}

The above-mentioned problems of the industry have led to the development of a so-called GEN IV reactors. They are described as radically different designs to reactors operating today, some involving a closed fuel cycle. Moreover, GEN IV reactors are said to be highly economical, to incorporate enhanced safety and reliability features and embed physical protection. They should produce minimal amounts of waste and be proliferation resistant, as these designs include advanced actinide management.

In addition, GEN IV reactors should have all of the features of Gen III+ units, as well as the ability, when operating at high temperature, to support economical hydrogen production, thermal energy off-taking, and perhaps even water desalination.

Yet, today, in 2018, GEN IV reactors are considered to be decades away from commercialization, as they have been when the discussion on these concepts started again in the early 2000s. The few attempts in the past based on concepts like fast neutron reactors nowadays called GEN IV ended with severe technical issues (e.g. Phénix and Superphénix in France) or accidents (e.g. Monju in Japan), which forced a closure of the respective project. Only two are currently in operation (Beloyarsk-3 and -4).

The start of the GEN IV initiative can be pointed to the year 2000, when the U.S. DOE launched the "Generation IV International Forum" (GIF) with the aim of developing a technically new generation of reactors. Today, ten member-countries participate in this initiative (Argentina, Brazil, Canada, France, Japan, Republic of Korea, South Africa, Switzerland, U.K. and the USA), as does EURATOM. The initial goal was to develop innovative nuclear systems (reactors and fuel cycles) likely to enter markets by as early of 2020 .

The concepts selected for Generation IV were discussed within the GIF groups of international experts from industry, universities and national laboratories. They were organized to undertake the identification and evaluation of candidate systems, and to define research and development (R\&D) activities to support them. Initially, some 100 different designs were identified as candidates and evaluated.

These designs ranged from concepts that rather belonged to Generation III+ to a few that were radically different from all known technologies. At the end of the process, six concepts were recommended for further development in the framework 
of GIF. A roadmap describes the R\&D required to develop each of the six systems as well as the approximate time and cost for completion. Many of the technological gaps were common to more than one system and the roadmap identifies several areas where crosscutting $R \& D$ would be required. The necessary $R \& D$ would be very expensive, and no single country had the necessary facilities and expertise to carry it out alone (DOE, 2002).

\subsection{Sodium-Cooled Fast Reactor System (SFR)}

The SFR system consists of a fast-neutron reactor and a closed fuel cycle system. There are two major options: One is a medium size (150 to $500 \mathrm{MWe}$ ) reactor with metal alloy fuel, supported by a fuel cycle based on pyrometallurgical reprocessing in collocated facilities. The second is a medium to large (500 to 1,500 MWe) reactor with MOX fuel, supported by a fuel cycle based upon advanced aqueous reprocessing at a centralized location serving a number of reactors. The primary coolant system can either be arranged in a pool layout or in a compact loop layout. (DOE, 2002; Lineberry, 2002).

According to GIF, the SFR has the broadest development base of all the GEN IV concepts. The existing know-how, however, is based mainly on old reactors, which have already been shut down for various reasons (safety, economics, resistance from the population), e.g Superphenix in France (1988) and Kalkar in Germany (1989).

Considering its history, as well as the significant hazards of this reactor line it is difficult to understand, why the SFR has been selected by GIF. According to GIF, research on both, the fuel cycle and the reactor system, is necessary to bring the SFR to deployment. Furthermore, there is important work to be done regarding safety. It has been argued that the SFR system is top ranked in sustainability because of its closed fuel cycle and potential for actinide management. It has been rated good in safety, economics, proliferation resistance and physical protection. In 2002 the SFR system was estimated to be deployable by 2015 (DOE, 2002). It did not happen.

\subsection{Very-High-Temperature Reactor System (VHTR)}

The VHTR is considered as a next step in the evolutionary development of high-temperature gas-cooled reactors (HTGR). This reactor line has been pursued until the late 80 s in several countries; however, only prototype and demonstration plants were ever operated, all of which were decommissioned after rather short and unsuccessful overall operating times about twelve years of operation at most such 
as the small Dragon reactor experiment (20 MWth, 1966 -1975, U.K.), the THTR Hamm-Uentrop, (308 MWe, 1986-1988, Germany) as well as the U.S. plants at Peach Bottom (42 MWe, 1967-1974) and Fort St. Vrain (342 MWe, 1976-1989).

The VHTR system uses a thermal neutron spectrum and a once-through uranium fuel cycle. The reference reactor concept has a 600-MWth graphite-moderated helium-cooled core based on either the prismatic block fuel of the GT-MHR or the pebble bed of the PBMR. It is regarded as the most promising and efficient system for hydrogen production, either using the thermochemical iodine-sulphur process, or from heat, water, and natural gas by applying the steam reformer technology at core outlet temperatures greater than $1,000^{\circ} \mathrm{C}$. The VHTR is also intended to generate electricity with high efficiency (over 50\%).

Furthermore, it is hoped that the concept could benefit from the experience gained with the Japanese HTTR research reactor and the Chinese HTR-PM still under construction, as well as from the GT-MHR and the PBMR projects at present in the planning phase.

\subsection{Supercritical-Water-Cooled Reactor System (SCWR)}

The SCWRs are high-temperature, high-pressure water-cooled reactors that operate above the thermo-dynamic critical point of water $\left(\mathrm{T}>374,12^{\circ} \mathrm{C}\right.$ and $\left.\mathrm{p}>22,06 \mathrm{GPa}\right)$. The reference plant has a $1700 \mathrm{MWe}$ power level, an operating pressure of $25 \mathrm{MPa}$, and a reactor outlet temperature of $550^{\circ} \mathrm{C}$. Fuel is uranium oxide. SCWRs could be designed as thermal or as fast-spectrum reactors, but current worldwide efforts focus on the thermal design.

The thermal efficiency of a SCWR can approach $44 \%$, compared to $33-35 \%$ for LWRs. Because no change of phase occurs in the core and the system utilizes a direct cycle (like the BWR), steam separators, dryers, pressurizes and recirculation pumps are not required, resulting in a considerably simpler and more compact system than traditional LWRs. SCWRs are hoped to be more economical than LWRs, due to plant simplification and high thermal efficiency. The Governments of Japan, the U.S. and Canada are developing the SCWR. There have been no prototypes built so far.

The technology for the SCWR is based on the existing LWRs and supercritical-water-cooled fossil-fired power plants. However, there are important SCWR technology gaps in the areas of materials and structures, including corrosion and stress corrosion cracking (SCC), safety and plant design. The main feasibility issues are the development of suitable in-core materials and the demonstration of adequate safety and stability. 


\subsection{Lead-Cooled Fast Reactor System (LFR)}

LFR systems are reactors cooled by liquid metal (lead or lead/bismuth) with a fast-neutron spectrum and closed fuel cycle system. A full actinide-recycle fuel cycle with central or regional facilities is envisaged. A wide range of unit sizes is planned, from 'batteries' of 50-150 MWe, and modular units of 300-400 MWe to large single plants of $1200 \mathrm{MWe}$. The LFR battery option is a small factory-built turnkey plant with very long core life (10 to 30 years). It is designed for small grids, and for developing countries that may not wish to deploy a fuel cycle infrastructure.

Among the LFR concepts, this battery option is regarded as the most promising, concerning fulfilment of Generation IV goals. However, it also has the largest research needs and longest development time.

Experience with the technology is restricted to seven Russian Alpha class submarines, which stopped operation in 1995, and on the advanced liquid-metal fast breeder reactor (ALMR), the design of which was withdrawn from the U.S. NRC review at an early stage (WANO, 2004).

The LFR system is top-ranked in sustainability because a closed fuel cycle is aimed at, and in proliferation resistance and physical protection because it employs a long-life core. It is rated good in safety and economics. The LFR system was estimated in 2002 to be deployable by 2025 (DOE, 2002). That is out of reach today.

\subsection{Gas-Cooled Fast Reactor System (GFR)}

The GFR system is a helium-cooled reactor with fast-neutron spectrum and closed fuel cycle. It is primarily envisioned for electricity production and actinide management. The GFR reference assumes an integrated, on-site spent fuel treatment and re-fabrication plant, but the viability of the planned technology has yet to be demonstrated. Fuel cycle technology is the most comprehensive technology gap of the GFR.

In spite of large technology gaps, according to GIF, the GFR system is topranked in sustainability because of its closed fuel cycle and excellent theoretical performance in actinide management. It is rated good in safety, economics, as well as proliferation resistance and physical protection. The GFR was estimated in 2002 to be deployable by 2025 (DOE, 2002). That will not happen.

Several GIF members have a specific interest for a sequenced development of gas-cooled system: The first step of the 'Gas Technology Path ' aims to develop a modular HTGR, the second step would be the VHTR, and the third step the GFR 
(Carré, 2004). The gas-cooled systems VHTR and GFR are seen as the top priorities of GIF members in Europe and the USA.

\subsection{Molten Salt Reactor System (MSR)}

During the 1960s the USA developed the molten salt breeder reactor as the primary back-up option for the conventional fast breeder reactor (cooled by liquid metal). A small prototype (8 MWth), the Molten Salt Reactor Experiment (MSRE), was operated for only four years. The next project planned, the Molten Salt Breeder Reactor (MSBR), was never built. The present work rests only on these projects. Detailed designs of an MSR have not been produced since the 1970s (Forsberg, 2002).

The MSR system is based on a thermal neutron spectrum and a closed fuel cycle. The uranium fuel is dissolved in the sodium fluoride salt coolant that circulates through graphite core channels. The heat, directly generated in the molten salt, is transferred to a secondary coolant system, and then through a tertiary heat exchanger to the power conversion system.

\subsection{Discussion of GEN IV concepts}

GIF considers a closed fuel cycle as a major advantage of Generation IV concepts. A system with a closed fuel cycle is regarded as more effective, and sustainable. However, not all of the six concepts selected for development employ a closed fuel cycle. The VHTR, most favoured, relies on an open cycle; and for the SCWR, once-through constitutes the nearer-term option. Furthermore, it is questionable, whether it will actually be possible to successfully develop and implement a closed fuel cycle. In addition, the costs of such closed fuel cycle concepts would be very high. According to the study "The Future of Nuclear" of the U.S. Massachusetts Institute of Technology (MIT, 2003), a convincing case has not yet been made that the long-term waste management benefits of advanced closed fuel cycles involving reprocessing of spent fuel are not indeed outweighed by the short-term risks and costs, including proliferation risks. The waste problem of nuclear industry can only be reduced even in an optimistic closed cycle scenario but remains far from being solved. (e.g. Gutachten Transmutation, Gerald Kirchner et al., 2015) Also, the MIT study found that the fuel cost with a closed cycle, including waste storage and disposal charges, to be about 4.5 times the cost of a once-through cycle. Therefore, it is not realistic to expect that there will be new reactor and fuel cycle technologies that simultaneously overcome the problems of cost, safe waste disposal 
and proliferation. As a result, the MIT study concludes that the once-through fuel cycle best meets the criteria of low costs and proliferation-resistance (NEI, 2003).

The basic concepts of the "new generation" have been around as long as nuclear power, but they were forced out of the market in the early years by the LWR - not without reason, considering the experiences so far, which are dominated by technical and economic problems, and safety deficits.

In order to overcome these problems, materials, processes and operating regimes that are significantly different from those of currently operating systems or previous systems would have to be developed. So far, none of the six reactor concepts selected for development fulfils all GEN IV aims.

\section{Conclusions}

Practically all of the 413 world-wide operating reactors (as of mid-2018) are GEN-II reactors carrying the well-known risks with respect to safety issues and proliferation. By the end of 2018, only a hand full of GEN III+ reactors - attributed with higher safety levels - are in operation (all in China). There are only 11 are under construction world-wide. They should be safer due to additional passive safety features. The lessons learned so far are that these generations' plants are much more expensive and can experience huge delays in construction times.

Different international, government sponsored organisations such as GIF are selling the idea of so-called GEN IV reactors. They pretend that under that umbrella term a completely new generation of reactors is being developed. The envisaged concepts are surely different from the light water concepts used so far. The message for the media, politicians and the public is: GEN IV is a safe, economical competitive, proliferation-resistant power source without the problem of increasing greenhouse gas emissions.

However, these expectations are very high, and their realization is at least 20 years away. In addition, the underlying basic concepts of "GEN IV" are decades old and encompass a range of ideas, which were already discussed in the 1950s. They were not pursued further due to severe problems such as high costs, limiting factors in material properties and difficulties in controlling the fission processes. They were forced out of the market already in the early years by the LWR.

It is of core interest to understand what the real motivation behind the GEN IV initiative is. To some extent it seems the revival of the discussion on GEN IV is mainly driven by the wish to secure the flow of public money for nuclear research. Whether it will really lead to the development of new reactors remains highly 
doubtful. The sobering final conclusion is that substantial amounts of money are invested in efforts which are not likely to solve any of the problems of nuclear power, climate change and energy supply security.

\section{References}

Carré, 2004: R\&D Program on Generation IV Nuclear Energy System: The High Temperature Gas-cooled Reactors; Annual Meeting on Nuclear Technology, 2004, May 25-27, Düsseldorf

DOE, 2002: United States Department of Energy's the Generation IV International Forum (GIF): A Technology Roadmap for Generation IV Nuclear Energy System; December 2002, (http://energy.inel.gov/gen-iv/docs/gen_iv_roadmap.pdf )

Forsberg, 2002: Molten Salt Reactors (MSRs); Americas Nuclear Energy Symposium (ANES 2002), Miami, Florida; October 16-18, 2002

Goldberg S. M., Rosner R., 2011. Nuclear reactors: Generation to Generation, American Academy of Arts and Sciences.

Greenpeace, 2005. Nuclear Reactor Hazards: Ongoing Dangers of Operating Nuclear Technology in the 21st Century. April 2005.

IAEA, 2004: International Atomic Energy Agency: Nuclear Technology Review 2004; Vienna, August 2004

Lineberry, 2002: Lineberry, M. J.; Allen T. R:. The Sodium-Cooled Fast Reactor (SFR); Americas Nuclear Energy Symposium (ANES 2002), Miami, Florida; October 16-18, 2002

MIT, 2003:An Interdisciplinary MIT Study: John Deutch (Co-Chair), Ernest J. Moniz (CoChair), Stephen Ansolabehere, Michael Driscoll, Paul E. Gray, John P. Holdren, Paul L. Joskow, Richard K. Lester, and Neil E. Todreas; The future of nuclear power, January 2003, Massachusetts Institute of Technology

NEI,2003: Nuclear Engineering International: The future lies in the past; October 2003, 42-45

WNA, 2004: World Nuclear Association: Advanced Nuclear Power Reactors; November 2004, www.world-nuclear.org)

WNISR, 2018. The World Nuclear Industry Status Report 2018.

Open Access This chapter is licensed under the terms of the Creative Commons Attribution 4.0 International License (http://creativecommons.org/licenses/by/4.0/), which permits use, sharing, adaptation, distribution and reproduction in any medium or format, as long as you give appropriate credit to the original author(s) and the source, provide a link to the Creative Commons license and indicate if changes were made.

The images or other third party material in this chapter are included in the chapter's Creative Commons license, unless indicated otherwise in a credit line to the material. If material is not included in the chapter's Creative Commons license and your intended use is not permitted by statutory regulation or exceeds the permitted use, you will need to obtain permission directly from the copyright holder.

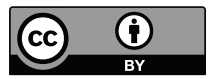

